\title{
Rapid Weight Loss vs. Slow Weight Loss: Which is More Effective on Body Composition and Metabolic Risk Factors?
}

\author{
Damoon Ashtary-Larky, ${ }^{1,2}$ Matin Ghanavati, ${ }^{3}$ Nasrin Lamuchi-Deli, ${ }^{2}$ Seyedeh Arefeh Payami, ${ }^{2}$ Sara \\ Alavi-Rad, ${ }^{2}$ Mehdi Boustaninejad, ${ }^{4}$ Reza Afrisham, ${ }^{5}$ Amir Abbasnezhad, ${ }^{7}$ and Meysam Alipour ${ }^{2,6,{ }^{*}}$ \\ ${ }^{1}$ Department of Clinical Biochemistry, School of Medicine, Ahvaz Jundishapur University of Medical Sciences, Ahvaz, Iran \\ ${ }^{2}$ Student Research Committee, Ahvaz Jundishapur University of Medical Sciences, Ahvaz, Iran \\ ${ }^{3}$ Department of Clinical Nutrition and Dietetics, Faculty of Nutrition and Food Technology, Shahid Beheshti University of Medical Sciences, Tehran, Iran \\ ${ }^{4}$ Department of Physical Education and Sports Sciences, Central Tehran Branch, Islamic Azad University, Tehran, Iran \\ ${ }^{5}$ Department of Clinical Biochemistry, School of Medicine, Tehran University of Medical Sciences, Tehran, Iran \\ ${ }^{6}$ Nutrition and Metabolic Diseases Research Center, Ahvaz Jundishapur University of Medical Sciences, Ahvaz, Iran \\ ${ }^{7}$ Nutritional Health Research Center, Department of Nutrition, Lorestan University of Medical Sciences, Khorramabad, Iran \\ "Corresponding author: Meysam Alipour, Nutrition and Metabolic Diseases Research Center, Ahvaz Jundishapur University of Medical Sciences, P.O.BOX:159613- 5715794, Ahvaz, \\ Iran. Tel: +98-6133720299, E-mail: meysam.aalipour@yahoo.com
}

Received 2016 November 16; Revised 2017 March 03; Accepted 2017 March 22.

\begin{abstract}
Background: Achieving weight loss (WL) in a short time regardless of its consequences has always been the focus of many obese and overweight people. In this study, anthropometric and metabolic effects of two diets for rapid and slow WL and their consequences were examined.

Methods: Forty-two obese and overweight individuals were randomly divided to 2 groups; rapid WL (weight loss of at least $5 \%$ in 5 weeks) and slow WL (weight loss of at least $5 \%$ in 15 weeks). To compare the effects of the rate of WL in 2 groups, the same amount of was achieved with different durations. Anthropometric indices, lipid, and glycemic profiles, and systolic and diastolic blood pressures were evaluated before and after the intervention.

Results: Both protocols of rapid WL and slow WL caused reduction in waist circumference, hip circumference, total body water, body fat mass, lean body mass, and resting metabolic rate (RMR). Further reduction in waist circumference, hip circumference, fat mass, and percentage of body fat was observed in slow WL and decreased total body water, lean body mass, fat free mass, and RMR was observed in rapid WL. Improvement in lipid and glycemic profiles was observed in both groups. Reduction of low-density lipoprotein and fasting blood sugar, improvement of insulin resistance, and sensitivity were more significant in rapid WL in comparison to slow WL.

Conclusions: Weight Loss regardless of its severity could improve anthropometric indicators, although body composition is more favorable following a slow WL. Both diets improved lipid and glycemic profiles. In this context, rapid WL was more effective. (IRCT2016010424699N2)
\end{abstract}

Keywords: Body Composition, Diet, Insulin Resistance, Obesity, Weight Loss

\section{Background}

The prevalence of obesity is increasing and according to the latest statistics of the world health organization, $13 \%$ of adults worldwide are obese and 39\% are overweight (1). Obesity increases the risk of metabolic diseases, cancer, and cataract $(2,3)$. Statistics have shown that obesity and its consequences have high costs for communities (4). There is a positive association between body mass index (BMI) and direct and indirect (due to premature deaths) health care costs. Indirect costs of obesity (54\% to $59 \%$ ) have been reported more than the direct costs (5). In the last century, due to the increasing prevalence of obesity and its hidden costs, control and treatment of obesity requires more attention.

Weight loss (WL) in obese patients, in addition to im- proving clinical conditions, will increase the recognition and quality of life $(6,7)$. In order to lose weight, various methods, such as diet, physical activity, drug therapy, and surgery have been suggested. Given the potential side effects of drug therapy and surgery, dietary interventions for WL have always been the first priority for the subjects (8). However, a variety of diets for WL have been suggested.

The difference in body composition (muscle loss and dehydration), metabolic effects, and the return of weight has been reported $(9,10)$. In a meta-analysis study, weight return had been reported in most participants (77\%), who followed WL diets (11). In a classification of diets based on calorie restrictions and speed of $\mathrm{WL}$, diets are divided to rapid WL, moderate $\mathrm{WL}$, and slow $\mathrm{WL}$.

Although many studies recommended gradual WL di- 
ets for obese patients, many people would like to lose their excess weight in the shortest time (12). A significant number of people believe that rapid WL has side effects and cannot have beneficial clinical effects similar to slow WL. However, a systematic review found that people, who follow severe calorie-restricted diets will not have an eating disorder and will be able to maintain their lost weight (13). Several studies have shown that rapid WL with high calorierestriction could cause an improvement of clinical state in obese individuals $(14,15)$.

Harder et al. reported that rapid WL could significantly decrease weight, triglycerides, total cholesterol(TC), low-density lipoprotein (LDL) cholesterol, fasting blood glucose (FBS), hemoglobin A1c, and fasting serum insulin (FINS) (16). Also, Wahlroos et al. reported that a significant decrease in waist circumference, body mass index (BMI), subcutaneous abdominal fat volume, and insulin resistance occurred after rapid WL (17).

However, it seems that the effects of metabolic and anthropometric from slow WL are different from rapid WL. In a pilot study, the difference between these 2 diets on anthropometric status was reported (18). Also, Yudai et al. showed that body weight and total intra-abdominal fat mass in the rapid and slow WLs decreased to the same extent, yet muscle atrophy was significantly higher with rapid than slow WL (19). The review of studies showed that metabolic differences of these 2 types of diets are still unclear.

\section{Objectives}

The aim of this clinical trial study was to evaluate the effects of glycemic and lipid parameters of the two protocols on WL in obese and overweight people.

\section{Methods}

This double-blind clinical trial study was conducted on 42 obese and overweight individuals $(25<\mathrm{BMI}<35)$. Participants were selected from those, who referred to a nutrition clinic (Ahvaz, Iran). Participants were screened based on the inclusion and exclusion criteria. Inclusion criteria were lack of physical activity, no smoking, no alcohol drinking, no usage of herbal supplements and vitamins, and lack of weight changes in the last 6 months. Exclusion criteria included pregnancy, breastfeeding, use of drugs that effect metabolism, lipid and glycemic profile, eating disorder, diabetes, cardiovascular disease, kidney problems, thyroid, digestive, respiratory diseases, and cancer. Participants consuming more than $300 \mathrm{mg}$ of caffeine daily (described as caffeine users) were excluded from the study (20). The level of physical activity was assessed weekly by phone. The subjects, who had moderate or various physical activities, were excluded from the study.

At the beginning, individuals were selected from the nutrition clinic. The initial screening had been done after a brief explanation of the study, and preliminary evaluation was done by phone. Next, a meeting with complete description of the protocol and justification for the study was arranged for the volunteers. The final screening was carried out in accordance with the inclusion and exclusion criteria. Eligible individuals, after filling the consent form, were randomly divided to 2 groups, rapid WL and slow WL.

Prior to WL, an ambulatory run-in period was imposed for each subject to insure stabilization of body weight ( \pm $2 \mathrm{~kg}$ during 4 weeks). During the body weight stabilization, a three-day food dietary record was used to determine an individual's daily food and beverage consumption to estimate their total daily caloric intake (2 weekdays and 1 weekend day). The subjects were randomly divided (according to age, gender and BMI) into two groups (rapid WL and slow WL). Rapid WL and slow WL, based on the lost weight (at least $5 \%$ ), were defined over a period of 5 weeks and 15 weeks, respectively (18). The prescribed calorie-restricted diet contained 15\% protein, 30\% to $35 \%$ fat, and $50 \%$ to $55 \%$ carbohydrate, on average, in order to provide WL. In general, the meal plans included 3 main meals (breakfast, lunch, and dinner) and three snacks (mid-morning, mid-afternoon, and bedtime), and low saturation and trans fats, cholesterol, salt (sodium), and added sugars. All diets were designed according to Dietary Guidelines for Americans, 2010 (21). Low-calorie diets produced an energy deficit of 500 to 750 and 1000 to 1500 kcal per day for slow and rapid WL, respectively. At the end of the study, anthropometric and biochemical assessments were conducted on the individuals (18 individuals in rapid WL and 18 individuals in slow WL), who reached the desired WL. All subjects provided their written informed consent, and the study protocol was approved by the ethics committee of Jundishapur University of Medical Sciences (Act No. IR.AJUMS.REC.1394.212).

Body weight and body composition were measured using the direct segmental multi-frequency bioelectrical impedance method (Inbody 230, Biospace, Korea) (22). The measurements presented were fasting state, shortly after waking in the morning, and at a dehydrated state. Standing height without shoes was measured using a stadiometer. Body Mass Index was calculated with the following formula: weight $(\mathrm{kg}) /$ height $^{2}\left(\mathrm{~m}^{2}\right)$. Waist circumference was obtained at the level of the noticeable waist narrowing, located approximately half way between the costal border and the iliac crest and the level of the greatest poste- 
rior protuberance. Hip circumference was also measured in the region of the greatest posterior protuberance and at approximately the symphysion pubis level, anteriorly. Blood pressure was measured using an automatic blood pressure monitor (BM65, Beurer, Germany) after subjects rested for more than 10 minutes. All anthropometric and blood pressure measurements were done in triplicates and the mean was calculated for each subject. Resting metabolic rate was measured at baseline and following the dietary intervention by indirect calorimetry (FitMate, Cosmed, Rome, Italy), using resting oxygen uptake (VO2).

Blood samples $(5 \mathrm{~mL})$ were collected at the beginning and at end of the study during the 12-hour fasting condition. The samples were centrifuged at a low level and serum was separated. Biochemical measurements were performed immediately after sampling. Fasting blood sugar (FBS), high-density lipoprotein (HDL), very low-density lipoprotein (VLDL), triglycerides (TG), and TC were measured by an auto-analyzer (Hitachi, USA). The Friedewald formula was used to calculate LDL levels. Fasting serum insulin concentration was measured by enzyme linked immunosorbent assay (ELISA) kits (Mercodia). The homeostatic model assessment (HOMA) was calculated with the formula: HOMA-IR $=\left[\right.$ FBS $(\mathrm{mg} / \mathrm{dL})^{*}$ FINS $\left.(\mu \mathrm{U} / \mathrm{mL})\right]$ | 405. (23). Quantitative insulin sensitivity check index (QUICKI) was calculated on the basis of suggested formulas: 1 / [log (Insulin $\mu \mathrm{U} / \mathrm{mL}$ ) + log (Glucose mg/dL)]. (24). The HOMA-B (pancreatic beta cell function) was computed as follow: $20 \times$ FINS $(\mu \mathrm{IU} / \mathrm{mL}) /$ fasting glucose $(\mathrm{mmol} / \mathrm{mL})$ 3.5. Insulin sensitivity was derived using the formula: HOMA-S (insulin sensitivity) $=22.5 /($ insulin $(\mathrm{mU} / \mathrm{L}) \times$ glucose $(\mathrm{mmol} / \mathrm{L}))$. All biochemical assays were performed in duplicates and the mean was calculated for each subject.

Statistical analyses were conducted using SPSS version 19.0 (SPSS Inc., Chicago, IL, USA). The data were checked for normality using the Kolmogorov-Smirnov test. Independent sample t test (for normally distributed variables) and Mann-Whitney U test (for non-normally distributed variables) were used to compare baseline values between the 2 groups. Moreover, in order to assay differences before and after the intervention within groups, paired sample t test (for normally distributed variables) and Wilcoxon test (for non-normally distributed variables) were used. Data were reported as mean \pm standard error. P values of $<0.05$ were considered significant.

\section{Results}

Of the forty-two participants assigned to the trial, thirty-six subjects completed the study ( $n=18$ in slow WL group and $n=18$ in rapid WL group). During the study, 3 individuals in the rapid WL group (medication consumption and discontinued) and 3 in the slow WL group (disinclination, medication consumption, and migration) were excluded (Figure 1). No significant side effect in the two study groups was detected.

Baseline characteristics in rapid WL and slow WL groups are shown in Table 1 . No significant differences were observed in baseline characteristics between the 2 groups $(\mathrm{P}>0.05)$.

As shown in Table 2, WL is statically the same in both groups (-5.47 \pm 1.46 and $-5.12 \pm 1.12$ for slow and rapid WL, respectively, $\mathrm{P}>0.05)$. The results of body composition, systolic and diastolic blood pressure, and heart rate are given in Table 2. A significant reduction in anthropometric indices and RMR were detected in both groups. Significant differences in blood pressure and heart rate were seen in the 2 groups. Waist circumference and hip circumference in slow WL group had a significant reduction compared to the rapid WL group.

A significant reduction in body fat (fat mass (FM), Body fat percentage, Arm fat percentage, feet FM, feet fat percentage) was observed in the slow WL group compared to the rapid WL group. In addition, a significant reduction in lean mass (lean body mass (LBM), fat free mass (FFM), Trunk lean) and total body water and RMR was seen in the rapid WL group compared to the slow WL group.

The glycemic and lipid profiles are shown in Table 3. Triglyceride and VLDL levels and insulin indices (FINS, HOMA-IR, and HOMA-S) showed a significant decrease in both groups. In addition, QUICKI increased significantly in both groups, yet no significant differences were shown between the 2 groups. Although the level of FBS and FINS changed significantly, especially in the rapid WL group, the drop in HOMA-B was not statistically significant. A significant reduction in LDL, FBS, and TC was seen in the rapid WL group. In addition, a significant reduction in HOMAIR, HOMA-S, FBS, and LDL was seen in rapid WL group compared to the slow WL group.

\section{Discussion}

The results of the current study showed that both protocols of rapid WL and slow WL caused a reduction in waist circumference, hip circumference, total body water, body fat mass, FFM, LBM, and RMR. Greater reduction of waist circumference, hip circumference, and FFM was seen with slow WL and greater reduction of total body water, LBM, and RMR was seen with rapid WL. It seems that the effect of slow WL in maintaining body water and LBM (as a metabolic tissue) was more significant than rapid WL.

In previous studies, elevated ratio of myostatin-tofollistatin, as an indicator of skeletal muscle catabolism, 


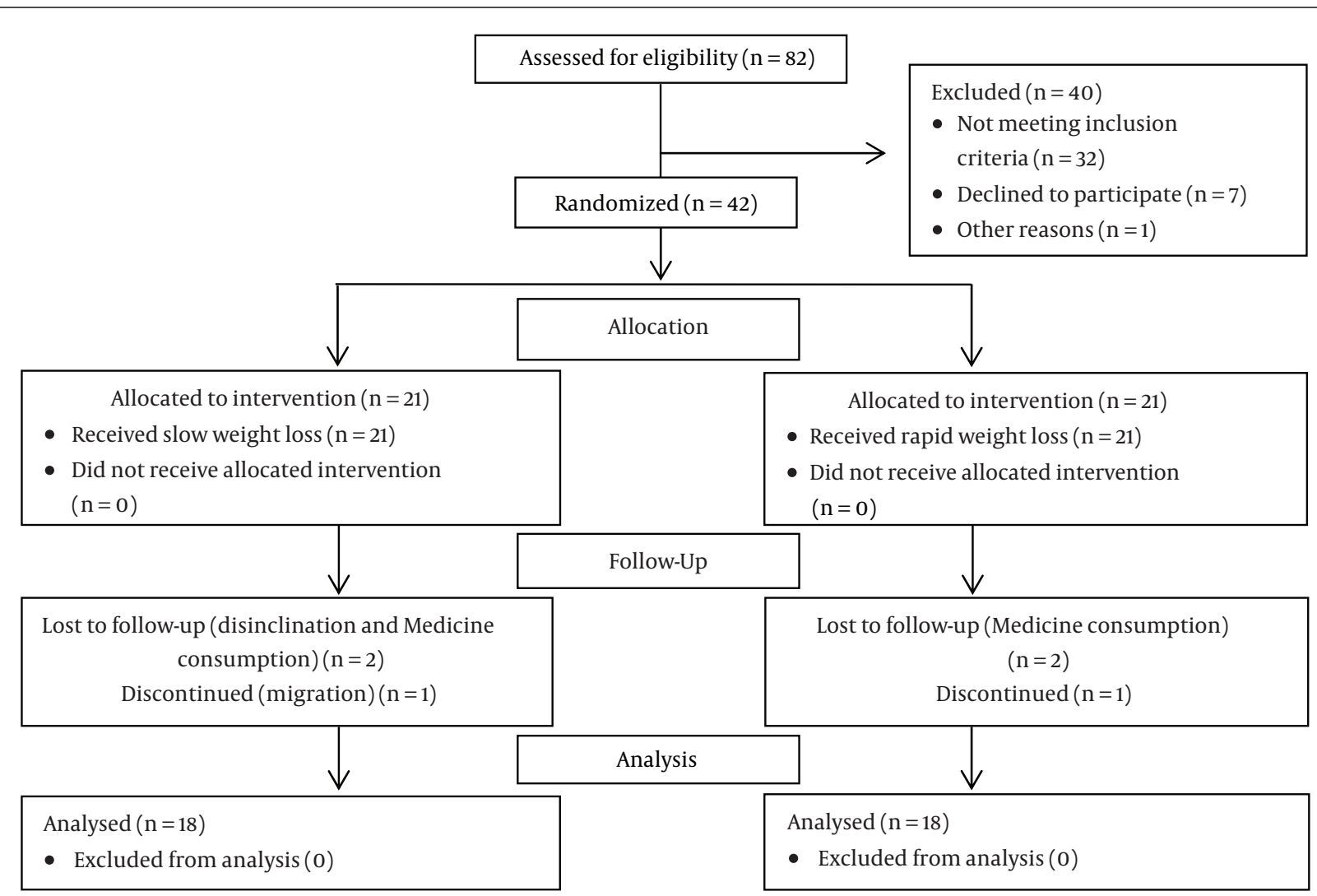

Figure 1. Flow Chart of the Study

Table 1. Baseline Characteristics of the Study in Both Groups ${ }^{\mathrm{a}}$

\begin{tabular}{|c|c|c|c|}
\hline Variables & Slow Weight Loss $(\mathrm{n}=18)$ & Rapid Weight Loss $(n=18)$ & P Value $^{\mathrm{b}}$ \\
\hline Age (years) & $37.1 \pm 11.2$ & $34.4 \pm 11.1$ & NS \\
\hline Female - N (\%) & $13(72.2 \%)$ & $13(72.2 \%)$ & NS \\
\hline Height (cm) & $161.4 \pm 7.7$ & $163.9 \pm 9.7$ & NS \\
\hline BMI $\left(\mathrm{kg} / \mathrm{m}^{2}\right)$ & $34.2 \pm 8.1$ & $32.0 \pm 6.3$ & NS \\
\hline PBF (\%) & $42.5 \pm 7.1$ & $39.1 \pm 10.2$ & NS \\
\hline SBP(mmHg) & $132.8 \pm 18.9$ & $133.0 \pm 17.4$ & NS \\
\hline $\mathrm{DBP}(\mathbf{m m H g})$ & $83.7 \pm 9.4$ & $82.7 \pm 8.7$ & NS \\
\hline Weight loss (\%) & $6.2 \pm 1.2$ & $6.0 \pm 0.8$ & NS \\
\hline
\end{tabular}

was reported to be greater in rapid WL compared to slow WL (25). The results were consistent with other studies in this field (26). In a study by Martin et al., the impact of these 2 protocols had been compared on the indices of anthro- pometric and lipid profiles. Their study was conducted in the form of a pilot study on obese postmenopausal females. The results of their study showed that slow WL caused more fat mass reduction and less FFM loss. How- 
Table 2. Anthropometric Status at Baseline and After the Intervention in Both Groups ${ }^{\mathrm{a}}$

\begin{tabular}{|c|c|c|c|c|c|c|c|}
\hline \multirow[t]{2}{*}{ Variables } & \multicolumn{2}{|c|}{ Slow Weight Loss $(n=18)$} & \multicolumn{2}{|c|}{ Rapid Weight Loss $(n=18)$} & \multicolumn{3}{|c|}{$\Delta$} \\
\hline & Baseline & End & Baseline & End & Intragroup $^{b}$ & Intragroup $^{c}$ & Intergroup $^{d}$ \\
\hline Weight (kg) & $86.9 \pm 16.1$ & $81.4 \pm 15.1$ & $85.5 \pm 15.3$ & $80.3 \pm 14.5$ & $-5.47 \pm 1.46^{\mathrm{e}}$ & $-5.12 \pm 1.12^{\mathrm{e}}$ & $0.35 \pm 0.43$ \\
\hline BMI $\left(\mathrm{kg} / \mathrm{m}^{2}\right)$ & $34.2 \pm 8.0$ & $32.1 \pm 7.6$ & $32.0 \pm 6.3$ & $30.0 \pm 6.0$ & $-2.10 \pm 0.52^{\mathrm{e}}$ & $-1.92 \pm 0.43^{\mathrm{e}}$ & $0.17 \pm 0.16$ \\
\hline$W C(\mathbf{c m})$ & $98.9 \pm 15.7$ & $92.8 \pm 15.0$ & $98.3 \pm 13.7$ & $93.7 \pm 13.2$ & $-6.09 \pm 1.60^{\mathrm{e}}$ & $-4.57 \pm 1.72^{\mathrm{e}}$ & $1.52 \pm 0.55^{\mathrm{f}}$ \\
\hline $\mathrm{HC}(\mathrm{cm})$ & $101.6 \pm 9.0$ & $96.5 \pm 8.4$ & $104.4 \pm 8.0$ & $101.2 \pm 7.4$ & $-5.07 \pm 1.10^{e}$ & $-3.11 \pm 2.15^{\mathrm{e}}$ & $1.95 \pm 0.57^{\mathrm{f}}$ \\
\hline WHR & $0.96 \pm 0.1$ & $0.95 \pm 0.1$ & $0.94 \pm 0.1$ & $0.92 \pm 0.1$ & $-0.01 \pm 0.01^{\mathrm{e}}$ & $-0.01 \pm 0.01^{\mathrm{e}}$ & $-0.001 \pm 0.001$ \\
\hline SBP (mmHg) & $132.5 \pm 18.6$ & $128.0 \pm 17.6$ & $133 \pm 17.4$ & $130.1 \pm 17.0$ & $-4.52 \pm 12.12$ & $-2.88 \pm 11.87$ & $1.63 \pm 4.00$ \\
\hline DBP (mmHg) & $83.7 \pm 9.4$ & $82.1 \pm 8.9$ & $82.7 \pm 8.7$ & $82.1 \pm 8.1$ & $-1.61 \pm 5.50$ & $-0.66 \pm 6.61$ & $0.94 \pm 2.02$ \\
\hline Heart rate & $97.3 \pm 15.7$ & $97.1 \pm 13.1$ & $96.8 \pm 15.0$ & $94.2 \pm 12.4$ & $-0.30 \pm 9.54$ & $-2.58 \pm 11.98$ & $-2.27 \pm 3.61$ \\
\hline RMR (kcal) & $1583.1 \pm 217$ & $1560.1 \pm 213.9$ & $1638.6 \pm 281.7$ & $1579.3 \pm 270.5$ & $-22.9 \pm 26.5^{\mathrm{f}}$ & $-59.3 \pm 32.6^{\mathrm{e}}$ & $-36.3 \pm 9.9^{e}$ \\
\hline LBM (kg) & $27.5 \pm 5.6$ & $27.0 \pm 5.6$ & $28.9 \pm 7.3$ & $27.4 \pm 7.0$ & $-0.52 \pm 0.75^{f}$ & $-1.51 \pm 0.80^{\mathrm{e}}$ & $-0.98 \pm 0.25^{\mathrm{e}}$ \\
\hline FM (kg) & $37.3 \pm 11.2$ & $32.8 \pm 10.9$ & $33.6 \pm 12.5$ & $30.7 \pm 11.9$ & $-4.52 \pm 1.71^{\mathrm{f}}$ & $-2.92 \pm 1.34^{\mathrm{e}}$ & $1.59 \pm 0.51^{\mathrm{f}}$ \\
\hline PBF (\%) & $42.5 \pm 7.1$ & $39.8 \pm 7.9$ & $39.1 \pm 10.2$ & $37.9 \pm 10.5$ & $-2.72 \pm 1.75^{\mathrm{f}}$ & $-1.15 \pm 1.44^{\mathrm{f}}$ & $1.57 \pm 0.53^{\mathrm{f}}$ \\
\hline TBW (kg) & $36.3 \pm 6.7$ & $35.5 \pm 6.6$ & $38.0 \pm 8.7$ & $36.3 \pm 8.4$ & $-0.72 \pm 0.85^{\mathrm{e}}$ & $-1.66 \pm 0.85^{\mathrm{e}}$ & $-0.93 \pm 0.28^{\mathrm{f}}$ \\
\hline FFM (kg) & $49.5 \pm 9.2$ & $48.5 \pm 9.9$ & $51.7 \pm 11.8$ & $49.5 \pm 11.2$ & $-0.98 \pm 1.17^{\mathrm{f}}$ & $-2.21 \pm 1.22^{\mathrm{e}}$ & $-1.23 \pm 0.40^{\mathrm{f}}$ \\
\hline Arm lean (kg) & $5.4 \pm 1.4$ & $5.3 \pm 1.3$ & $5.8 \pm 2.0$ & $5.6 \pm 1.8$ & $-0.10 \pm 0.27$ & $-0.25 \pm 0.53$ & $-0.15 \pm 0.14$ \\
\hline Trunk lean(kg) & $22.7 \pm 4.1$ & $22.4 \pm 4.0$ & $24.1 \pm 5.7$ & $23.1 \pm 5.5$ & $-0.29 \pm 0.75$ & $-1.01 \pm 0.51^{\mathrm{e}}$ & $-0.72 \pm 0.21^{\mathrm{f}}$ \\
\hline Feet lean (kg) & $14.9 \pm 3.0$ & $14.5 \pm 2.9$ & $15.4 \pm 3.4$ & $14.9 \pm 3.4$ & $-0.45 \pm 0.57^{\mathrm{f}}$ & $-0.47 \pm 0.35^{\mathrm{e}}$ & $-0.02 \pm 0.15$ \\
\hline $\operatorname{Arm}$ FM (kg) & $6.7 \pm 3.2$ & $5.3 \pm 2.7$ & $5.8 \pm 4.0$ & $5.0 \pm 3.5$ & $-1.46 \pm 1.42^{\mathrm{e}}$ & $-0.78 \pm 0.59^{\mathrm{e}}$ & $0.68 \pm 0.36$ \\
\hline Arm fat (\%) & $52.0 \pm 11.1$ & $47.7 \pm 12.2$ & $45.8 \pm 17.2$ & $43.9 \pm 17.2$ & $-4.25 \pm 2.62^{\mathrm{e}}$ & $-1.87 \pm 2.20^{\mathrm{f}}$ & $2.37 \pm 0.80^{\mathrm{f}}$ \\
\hline Trunk FM (kg) & $18.2 \pm 4.2$ & $16.5 \pm 4.7$ & $16.9 \pm 4.5$ & $15.5 \pm 4.7$ & $-1.72 \pm 1.35^{\mathrm{e}}$ & $-1.40 \pm 0.65^{\mathrm{e}}$ & $0.31 \pm 0.35$ \\
\hline Trunk Fat (\%) & $42.9 \pm 5.5$ & $40.6 \pm 6.6$ & $39.9 \pm 8.5$ & $38.6 \pm 9.0$ & $-2.27 \pm 1.89^{f}$ & $-1.25 \pm 1.37^{\mathrm{e}}$ & $1.02 \pm 0.55$ \\
\hline Feet FM (kg) & $10.9 \pm 3.8$ & $8.9 \pm 2.9$ & $9.5 \pm 4.0$ & $8.8 \pm 3.7$ & $-1.93 \pm 2.32^{\mathrm{f}}$ & $-0.65 \pm 0.54^{\mathrm{e}}$ & $1.28 \pm 0.56^{\mathrm{f}}$ \\
\hline Feet fat (\%) & $40.2 \pm 8.2$ & $37.5 \pm 8.3$ & $36.4 \pm 11.2$ & $35.4 \pm 11.3$ & $-2.70 \pm 1.49^{\mathrm{e}}$ & $-1.03 \pm 1.36^{f}$ & $1.67 \pm 0.47^{\mathrm{e}}$ \\
\hline
\end{tabular}

Abbreviations: BMI, body mass index; DBP, diastolic blood pressure; FFM, fat free mass; HC, hip circumference; LBM, lean boey mass; PBF, percent of body fat; SBP, systolic blood pressure; TBW, total body water; WC, waist circumference; WHR, waist-hip ratio.

${ }^{\mathrm{a}}$ All values are means $\pm \mathrm{SE}$.

${ }^{\mathrm{b}}$ Changes post-baseline in slow weight loss group.

${ }^{\mathrm{c}}$ Changes post-baseline in rapid weight loss group.

${ }^{\mathrm{d}}$ Changes between groups, for normally distributed variables, paired-sample t test and independent-sample t test were used to investigate differences within and between groups, respectively. For non-normally distributed variables, the Wilcoxon signed rank and Mann-Whitney U tests were used to assess differences within and between groups, respectively.

${ }^{\mathrm{e}}$ Significant differences were assumed at $\mathrm{P}<0.001$

${ }^{\mathrm{f}}$ Significant differences were assumed at $\mathrm{P}<0.05$.

ever, in their study, no differences in lipid profile were observed between slow WL and rapid WL (18).

No significant changes were observed in systolic and diastolic blood pressure in any of these 2 diets, although a non-significant reduction in average blood pressure at the end of the study was observed in both groups. It seems that the effects of weight-loss diets on the decrease of blood pressure was more concrete in people with hypertension (27). Consistent with the current study, several studies did not support the impact of WL on blood pressure in people, who had normal blood pressure $(16,28)$.

The current study showed that both protocols of WL could improve components of the lipid and glycemic profiles. In addition, in this study it was found that with the same amount of WL, the impact on reducing levels of FBS and LDL, and improvement of insulin resistance and sensitivity was greater with rapid WL. Positive effects of rapid WL on metabolic factors were reported in several studies.

Consistent with the current study, recent findings indicate that slow weight loss, as recommended by current 
Table 3. Lipid and Glycemic Profile at Baseline and After Six Weeks in Both Groups ${ }^{\mathrm{a}}$

\begin{tabular}{|c|c|c|c|c|c|c|c|}
\hline \multirow[t]{2}{*}{ Variables } & \multicolumn{2}{|c|}{ Slow Weight Loss $(n=18)$} & \multicolumn{2}{|c|}{ Rapid Weight Loss $(n=18)$} & \multicolumn{3}{|c|}{$\Delta$} \\
\hline & Baseline & End & Baseline & End & Intragroup $^{b}$ & Intragroup $^{c}$ & Intergroup $^{\mathrm{d}}$ \\
\hline LDL (mg/dL) & $105.9 \pm 34.3$ & $111.8 \pm 26.5$ & $120.9 \pm 28.7$ & $108.1 \pm 27.1$ & $5.91 \pm 30.95$ & $-12.8 \pm 14.4^{\mathrm{f}}$ & $-18.7 \pm 8.04^{\mathrm{f}}$ \\
\hline HDL (mg/dL) & $37.8 \pm 5.4$ & $39.7 \pm 6.2$ & $43.9 \pm 9.5$ & $45.1 \pm 6.3$ & $1.90 \pm 5.95$ & $1.16 \pm 6.08$ & $-0.73 \pm 1.99$ \\
\hline TG (mg/dL) & $145.3 \pm 65.8$ & $109.6 \pm 50.1$ & $163.8 \pm 70.5$ & $118.5 \pm 52.3$ & $-35.6 \pm 45.5^{\mathrm{f}}$ & $-45.3 \pm 56.8^{\mathrm{f}}$ & $-9.66 \pm 17.17$ \\
\hline $\operatorname{VLDL}(\mathbf{m g} / \mathbf{d L})$ & $29.0 \pm 13.1$ & $21.9 \pm 10.2$ & $32.7 \pm 14.1$ & $23.7 \pm 10.47$ & $-7.11 \pm 9.12^{\mathrm{f}}$ & $-9.05 \pm 11.37^{\mathrm{f}}$ & $-1.93 \pm 3.43$ \\
\hline $\mathrm{TC}(\mathbf{m g} / \mathbf{d L})$ & $172.4 \pm 40.5$ & $173.5 \pm 35.3$ & $194.2 \pm 39.6$ & $176.8 \pm 31.4$ & $1.06 \pm 33.64$ & $-17.4 \pm 19.6^{\mathrm{f}}$ & $-18.4 \pm 9.1$ \\
\hline FBS $(\mathrm{mg} / \mathrm{dL})$ & $95.1 \pm 11.1$ & $99.0 \pm 6.7$ & $100.8 \pm 18.8$ & $95.0 \pm 9.8$ & $3.92 \pm 10.36$ & $-5.87 \pm 11^{\mathrm{f}}$ & $-9.80 \pm 3.57^{\mathrm{f}}$ \\
\hline FINS $(\mu \mathbf{I U} / \mathbf{I})$ & $13.2 \pm 4.7$ & $11.5 \pm 5.4$ & $11.2 \pm 4.9$ & $7.6 \pm 4.3$ & $-1.96 \pm 2.52^{\mathrm{f}}$ & $-3.93 \pm 3.61^{\mathrm{e}}$ & $-0.06 \pm 1.96$ \\
\hline HOMA-IR & $1.7 \pm 0.6$ & $1.4 \pm 0.6$ & $1.5 \pm 0.7$ & $1.0 \pm 0.5$ & $-0.24 \pm 0.6^{\mathrm{f}}$ & $-0.43 \pm 0.46^{\mathrm{e}}$ & $-0.18 \pm 049^{f}$ \\
\hline HОМА-В & $126.1 \pm 26.2$ & $100.7 \pm 23.4$ & $106.6 \pm 50$ & $86.9 \pm 42.9$ & $-24.4 \pm 25.8$ & $-18.8 \pm 45.5$ & $5.36 \pm 41.2$ \\
\hline HOMA-S & $64.4 \pm 19.5$ & $79.3 \pm 29.6$ & $84.7 \pm 48.9$ & $131.7 \pm 67.7$ & $14.3 \pm 21.2^{\mathrm{e}}$ & $44.2 \pm 60.8^{\mathrm{f}}$ & $26.1 \pm 35.3^{\mathrm{f}}$ \\
\hline QUICKI & $0.32 \pm 0.01$ & $0.33 \pm 0.02$ & $0.33 \pm 0.02$ & $0.36 \pm 0.03$ & $0.01 \pm 0.01^{\mathrm{f}}$ & $0.03 \pm 0.03^{\mathrm{e}}$ & $1.16 \pm 6.08^{\mathrm{f}}$ \\
\hline
\end{tabular}

Abbreviations:TG, triglyceride; TC, total cholesterol; FBS, fasting blood sugar; FINS, fasting insulin; HOMA-IR, homeostasis model assessment insulin resistance; HOMA-B, HOMA-pancreatic beta cell function; HOMA-S, HOMA-insulin sensitivity; QUICKI, quantitative insulin sensitivity check index.

${ }^{a}$ All values are means \pm SD.

${ }^{\mathrm{b}}$ Changes post-baseline in slow weight loss group.

${ }^{\mathrm{C}}$ Changes post-baseline in rapid weight loss group.

${ }^{\mathrm{d}}$ Changes between groups, for normally distributed variables, paired-samples t test and independent-sample t-test were used to investigate the differences within and between groups, respectively. For non-normally-distributed variables, the Wilcoxon signed ranks test and a Mann -Whitney U test were used to assess differences within and between groups, respectively.

${ }^{\text {e}}$ Significant differences were assumed at $\mathrm{P}<0.001$.

${ }^{\mathrm{f}}$ Significant differences were assumed at $\mathrm{P}<0.05$.

guidelines, worldwide, is not a priority over rapid weight loss. Purcell et al. in a clinical trial studied the effect of weight loss rate and weight management. Their results showed that in the long-term, with rapid weight loss (450 to $800 \mathrm{Kcal} /$ day) than gradual weight loss (500 kcal less than the daily requirement), the weight loss is faster and more stable. The researchers suggested that the limited carbohydrate intake of very-low-calorie diets might promote greater satiety and less food intake by inducing ketosis. Losing weight quickly may also motivate participants to persist with their diet and achieve better results (29).

Evidence suggests that rapid weight loss through improvements in markers of oxidative stress could improve metabolic factors. Tumova et al. reported that rapid weight loss (800 kcal daily consisting of liquid beverages) could, through reducing oxidized low density lipoprotein (oxLDL) cause a decrease in total cholesterol. In addition, rapid weight loss could, through reducing the activity of Lipoprotein-associated phospholipase A2 (LP-PLA2), cause a decrease in levels of LDL-C, TC, and insulin in people with metabolic syndrome (30). Roberts et al. also reported that short-term diet (21-Day) and exercise intervention for males with metabolic syndrome factors could, through reducing level of inflammatory markers, such as myeloperoxidase (MPO), cause improvement in lipid risk factors and
HOMA-IR (30).

Consistent with the current study, improvement of metabolic factors after 4 weeks of VLCD was reported in a study by Erik et al. In this study, it was found that VLCD in the short-term intervention could cause a significant reduction in the levels of blood glucose, cholesterol, and TG in a fasting condition (27). The study of Laaksonen et al. showed that administration of VLCD diet for 5 weeks improved metabolic factors and decreased cutaneous water loss and increased subcutaneous fat water. This researcher suggested that WL and consequent improved insulin sensitivity could mediate an increase in abdominal subcutaneous fat hydration (31).

The results suggest that WL could improve anthropometric status and lipid and glycemic profiles regardless of calorie restriction and the speed of WL. However, there could be some differences between the 2 protocol types of WL in terms of impact. The WL regardless of its severity could improve anthropometric indicators, although body composition is more favorable following a slow WL. Both diets improved lipid and glycemic profiles. In this context, rapid WL was more effective.

Many studies have suggested that rapid weight loss may serve as a risk factor for later weight regain. Thus, a limitation of the current study was that it did not evaluate 
weight regain.

\section{Acknowledgments}

The authors wish to thank all patients, who participated in this research project.

\section{Footnotes}

Authors' Contribution: Study concept and design: Damoon Ashtary-Larky; field, experimental, and clinical work, and data collection: Damoon Ashtary-Larky, Nasrin Lamuchi-Deli, Mehdi Boustaninejad, Seyedeh Arefeh Payami, and Sara Alavi-Rad; data analysis and interpretation: Matin Ghanavati and Amir Abbasnezhad; preparation of the draft, revisions or providing critique: Meysam Alipour; overall and/or sectional scientific management: Reza Afrisham.

Conflicts of Interest: The authors declare no conflicts of interest.

\section{Funding/Support: No external sources of funding.}

\section{References}

1. Organization WH. . Obesity and overweight factsheet from the WHO. World; 2015.

2. Shahbazian H, Latifi SM, Jalali MT, Shahbazian H, Amani R, Nikhoo A, et al. Metabolic syndrome and its correlated factors in an urban population in South West of Iran.J Diabetes Metab Disord. 2013;12(1):11. doi: 10.1186/2251-6581-12-11. [PubMed: 23497506].

3. Ghanavati M, Behrooz M, Rashidkhani B, Ashtray-Larky D, Zameni SD, Alipour M. Healthy Eating Index in Patients With Cataract: A Case-Control Study. Iran Red Crescent Med J. 2015;17(10):22490. doi: 10.5812/ircmj.22490. [PubMed: 26568860].

4. Cawley J, Meyerhoefer C. The medical care costs of obesity: an instrumental variables approach. J Health Econ. 2012;31(1):219-30. doi: 10.1016/j.jhealeco.2011.10.003. [PubMed: 22094013].

5. Dee A, Kearns K, O’Neill C, Sharp L, Staines A, O’Dwyer V, et al. The direct and indirect costs of both overweight and obesity: a systematic review. BMC Res Notes. 2014;7:242. doi:10.1186/1756-0500-7-242. [PubMed: 24739239].

6. Napoli N, Shah K, Waters DL, Sinacore DR, Qualls C, Villareal DT. Effect of weight loss, exercise, or both on cognition and quality of life in obese older adults. Am J Clin Nutr. 2014;100(1):189-98. doi: 10.3945/ajcn.113.082883. [PubMed: 24787497].

7. Egert S, Baxheinrich A, Lee-Barkey YH, Tschoepe D, Wahrburg U, Stratmann B. Effects of an energy-restricted diet rich in plant-derived alpha-linolenic acid on systemic inflammation and endothelial function in overweight-to-obese patients with metabolic syndrome traits. Br J Nutr. 2014;112(8):1315-22. doi: 10.1017/S0007114514002001. [PubMed: 25180479].

8. Jakicic JM, Clark K, Coleman E, Donnelly JE, Foreyt J, Melanson E, et al. American College of Sports Medicine position stand. Appropriate intervention strategies for weight loss and prevention of weight regain for adults. Med Sci Sports Exerc. 2001;33(12):2145-56. [PubMed: 11740312].

9. Tay J, Luscombe-Marsh ND, Thompson $\mathrm{CH}$, Noakes M, Buckley JD, Wittert GA, et al. A very low-carbohydrate, low-saturated fat diet for type 2 diabetes management: a randomized trial. Diabetes Care. 2014;37(11):2909-18. doi: 10.2337/dc14-0845. [PubMed: 25071075].
10. Lasa A, Miranda J, Bullo M, Casas R, Salas-Salvado J, Larretxi I, et al. Comparative effect of two Mediterranean diets versus a low-fat diet on glycaemic control in individuals with type 2 diabetes. Eur J Clin Nutr. 2014;68(7):767-72. doi:10.1038/ejcn.2014.1. [PubMed: 24518752].

11. Anderson JW, Konz EC, Frederich RC, Wood CL. Long-term weightloss maintenance: a meta-analysis of US studies. Am J Clin Nutr. 2001;74(5):579-84. [PubMed: 11684524].

12. Pop LC, Sukumar D, Tomaino K, Schlussel Y, Schneider SH, Gordon CL, et al. Moderate weight loss in obese and overweight men preserves bone quality. Am J Clin Nutr. 2015;101(3):659-67. doi: 10.3945/ajcn.114.088534. [PubMed: 25733651].

13. da Luz FQ, Hay P, Gibson AA, Touyz SW, Swinbourne JM, Roekenes $J A$, et al. Does severe dietary energy restriction increase binge eating in overweight or obese individuals? A systematic review. Obes Rev. 2015;16(8):652-65. doi: 10.1111/obr.12295. [PubMed: 26094791].

14. Jazet IM, Schaart G, Gastaldelli A, Ferrannini E, Hesselink MK, Schrauwen $\mathrm{P}$, et al. Loss of $50 \%$ of excess weight using a very low energy diet improves insulin-stimulated glucose disposal and skeletal muscle insulin signalling in obese insulin-treated type 2 diabetic patients. Diabetologia. 2008;51(2):309-19. doi: 10.1007/s00125-007-08622. [PubMed: 18080107].

15. Madsen EL, Rissanen A, Bruun JM, Skogstrand K, Tonstad S, Hougaard DM, et al. Weight loss larger than $10 \%$ is needed for general improvement of levels of circulating adiponectin and markers of inflammation in obese subjects: a 3-year weight loss study. Eur J Endocrinol. 2008;158(2):179-87. doi:10.1530/EJE-07-0721. [PubMed: 18230824].

16. Harder H, Dinesen B, Astrup A. The effect of a rapid weight loss on lipid profile and glycemic control in obese type 2 diabetic patients. Int J Obes Relat Metab Disord. 2004;28(1):180-2. doi:10.1038/sj.ijo.0802529. [PubMed: 14610532].

17. Wahlroos S, Phillips ML, Lewis MC, Kow L, Toouli J, Slavotinek JP, et al. Rapid significant weight loss and regional lipid deposition: Implications for insulin sensitivity. Obes Res Clin Pract. 2007;1(1):1-78. doi: 10.1016/j.orcp.2006.08.002. [PubMed: 24351427].

18. Senechal M, Arguin H, Bouchard DR, Carpentier AC, Ardilouze JL, Dionne IJ, et al. Effects of rapid or slow weight loss on body composition and metabolic risk factors in obese postmenopausal women. A pilot study. Appetite. 2012;58(3):831-4. doi: 10.1016/j.appet.2012.01.014. [PubMed: 22306435].

19. Yudai N, Shin T. Differential effects of rapid or slow body weight loss on muscle weight and protein degradation pathways in rat skeletal muscle. J Int Soc Sports Nutr. 2015;12(1).

20. Shirali S, Daneghian S, Hosseini SA, Ashtary-Larky D, Daneghian M, Sadat Mirlohi M. Effect of caffeine co-ingested with carnitine on weight, body-fat percent, serum leptin and lipid profile changes in male teen soccer players: A randomized clinical trial. Inter J Pediatric. 2016;4(10):3685-98.

21. Services U, Agriculture U. Dietary guidelines for Americans, 2010. Washington, DC: US Government Printing Office; 2010.

22. Ghafourian M, Ashtary-Larky D, Chinipardaz R, Eskandary N, Mehavaran M. Inflammatory Biomarkers' Response to Two Different Intensities of a Single Bout Exercise Among Soccer Players. Iran Red Crescent Med J. 2016;18(2):21498. doi: 10.5812/ircmj.21498. [PubMed: 27175304].

23. Ghaffari MA, Payami SA, Payami SP, Ashtary-Larky D, Nikzamir A, Mohammadzadeh G. Evaluation of insulin resistance indices in type 2 diabetic patients treated with different anti-diabetic drugs. Open JEndocr Metab Dis. 2016;6(95).

24. Katz A, Nambi SS, Mather K, Baron AD, Follmann DA, Sullivan G, et al. Quantitative insulin sensitivity check index: a simple, accurate method for assessing insulin sensitivity in humans.J Clin Endocrinol Metab. 2000;85(7):2402-10. doi: 10.1210/jcem.85.7.6661. [PubMed: 10902785]. 
25. Motevalli MS, Dalbo VJ, Attarzadeh RS, Rashidlamir A, Tucker PS, Scanlan AT. The effect of rate of weight reduction on serum myostatin and follistatin concentrations in competitive wrestlers. Int J Sports Physiol Perform. 2015;10(2):139-46. doi: 10.1123/ijspp.2013-0475. [PubMed: 24911427].

26. Garthe I, Raastad T, Refsnes PE, Koivisto A, Sundgot-Borgen J. Effect of two different weight-loss rates on body composition and strength and power-related performance in elite athletes. Int J Sport Nutr Exerc Metab. 2011;21(2):97-104. [PubMed: 21558571].

27. Noren E, Forssell H. Very low calorie diet without aspartame in obese subjects: improved metabolic control after 4 weeks treatment. Nutr J. 2014;13:77. doi: 10.1186/1475-2891-13-77. [PubMed: 25069603].

28. Fujioka K, Seaton TB, Rowe E, Jelinek CA, Raskin P, Lebovitz HE, et al. Weight loss with sibutramine improves glycaemic control and other metabolic parameters in obese patients with type 2 diabetes mellitus.
Diabetes Obes Metab. 2000;2(3):175-87. [PubMed: 11220553].

29. Purcell K, Sumithran P, Prendergast LA, Bouniu CJ, Delbridge E, Proietto J. The effect of rate of weight loss on long-term weight management: a randomised controlled trial. Lancet Diabetes Endocrinol. 2014;2(12):954-62. doi: 10.1016/S2213-8587(14)70200-1. [PubMed: 25459211].

30. Tumova E, Sun W, Jones PH, Vrablik M, Ballantyne CM, Hoogeveen RC The impact of rapid weight loss on oxidative stress markers and the expression of the metabolic syndrome in obese individuals. J Obes. 2013.

31. Laaksonen DE, Nuutinen J, Lahtinen T, Rissanen A, Niskanen LK Changes in abdominal subcutaneous fat water content with rapid weight loss and long-term weight maintenance in abdominally obese men and women. Int J Obes Relat Metab Disord. 2003;27(6):677-83. doi 10.1038/sj.ijo.0802296. [PubMed: 12833111]. 